Int. J. Dev. Biol. 52: 1077-1088 (2008)

doi: $10.1387 /$ ijdb.082684hk

\title{
Spatio-temporal expression of a Netrin homolog in the sea urchin Hemicentrotus pulcherrimus (HpNetrin) during serotonergic axon extension
}

\author{
HIDEKI KATOW* \\ Research Center for Marine Biology, Tohoku University, Asamushi, Aomori, Japan
}

\begin{abstract}
A netrin homolog of the sea urchin, Hemicentrotus pulcherrimus (HpNetrin) was characterized in terms of its expression behavior. The predicted amino acid sequence was an ortholog of hemichordate netrin-1. Reverse transcriptase-PCR, immunoblotting, and whole mount immunohistochemistry showed that gene transcription and protein expression occurred from 15hour post-fertilization (hpf) swimming blastula stage to, at least 53-hpf pluteus stage. HpNetrin was detected on the entire basal surface of the ectoderm in swimming and 16-hpf mesenchyme blastulae. However, by 24-hpf prism stage, immunoreaction on the oral ectoderm decreased, whereas it increased in the aboral ectoderm including near the animal plate ectoderm (area-I). By 48-hpf pluteus stage, the HpNetrin-rich area-I comprised a $40 \mu \mathrm{m}$ wide dorsal midline belt (DMB) that stretched from the dorsal posterior edge of the animal plate to the posterior end of the larval body. Serotonergic cells were first detected in the HpNetrin-moderate area between the anterior DMB and the HpNetrin-poor oral ectoderm near the animal plate in 24-hpf prism larvae. By 48-hpf pluteus stage, these cells extended axons toward the middle-ridge to form a neural plexus of the apical ganglion. At 53-hpf pluteus stage, these axons extended further away from the apical ganglion directly or through the DMB toward the HpNetrin-poor contralateral ectoderm. The protein expression and axon extension pattern were reproduced in embryonic cell-aggregates formed from artificially dissociated 20-hpf gastrulae and resembled small blastula. In Hpnetrin morpholino antisense oligonucleotide-injected plutei, serotonergic axon extension was severely inhibited. Thus, HpNetrin functions as a serotonergic axon guidance cue in this basal deuterostome.
\end{abstract}

KEY WORDS: netrin, pathfinding, axon guidance, echinoplutei

\section{Introduction}

The first nervous system emerged during the early evolution of metazoan animals (Lichtneckert and Reichert, 2007). This system then flourished among bilaterians that, based on classical neuroanatomy, have been divided into two major groups: the Gastroneuralia that is characterized by a ventral central nervous system (CNS) and the Notoneuralia that is characterized by a dorsal CNS (Hirth and Reichert, 2007). Despite such evolutionally diverged CNS structures, their developmental mechanisms, such as axon guidance cues, are strikingly similar between them, implicating the presence of a common molecular bauplan underlining brain development (Kammermeier and Reichert, 2001). It has been increasingly clear that many molecular mechanisms of axon guidance are conserved to a remarkable degree amongst the Gastroneuralia and the Notoneuralia (Chisholm and TessierLavigne, 1999).

Sea urchins are basal deuterostomes and are crucial animal to understand the evolution of a Gastroneuralia type nervous system (see Byrne et al., 2007 for evolutionary view of the nervous system in basal deuterostomes). Unlike its adult form, the body plan of sea urchin embryos and larvae is bilateral, and highly motile as a result of ciliary beating on the body surface. The ciliary beating for swimming is under control of the serotonergic nervous system (Yaguchi and Katow, 2003; Katow et al., 2007) that is comprised both of a ganglion, in a small region of the apical most ectoderm (oral lobe ectoderm) (Nakajima et al., 1993; Yaguchi et

Abbreviations used in this paper: DMB, dorsal midline belt; $\mathrm{Hp}, H$. pulcherrimus; hpf, hours post fertilization; MASO, morpholino antisense oligonucleotide.

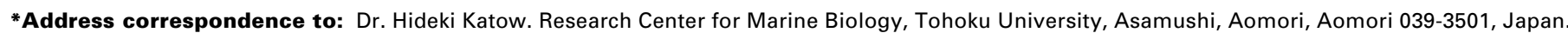
Tel. \& Fax: 81-17-752-3390. e-mail: hkatow@m.tains.tohoku.ac.jp
} 

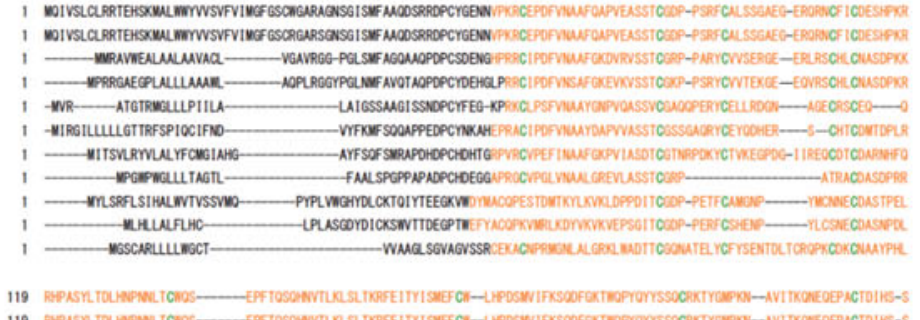
119 -

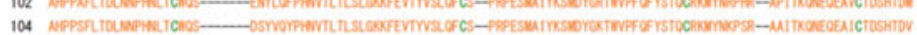

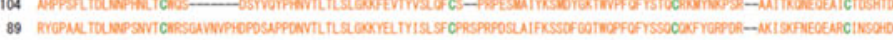

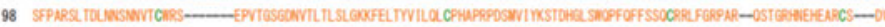

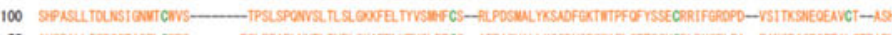

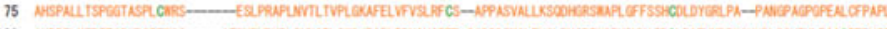

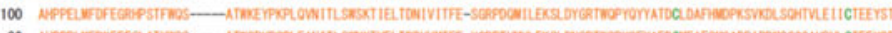

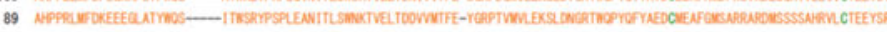

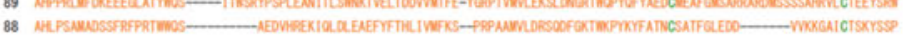

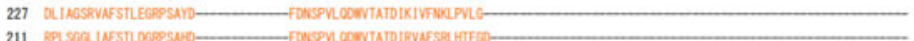

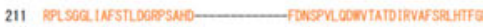

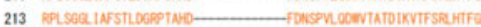

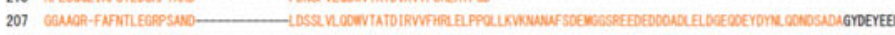

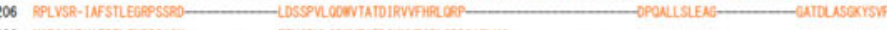

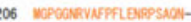

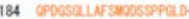

214

203
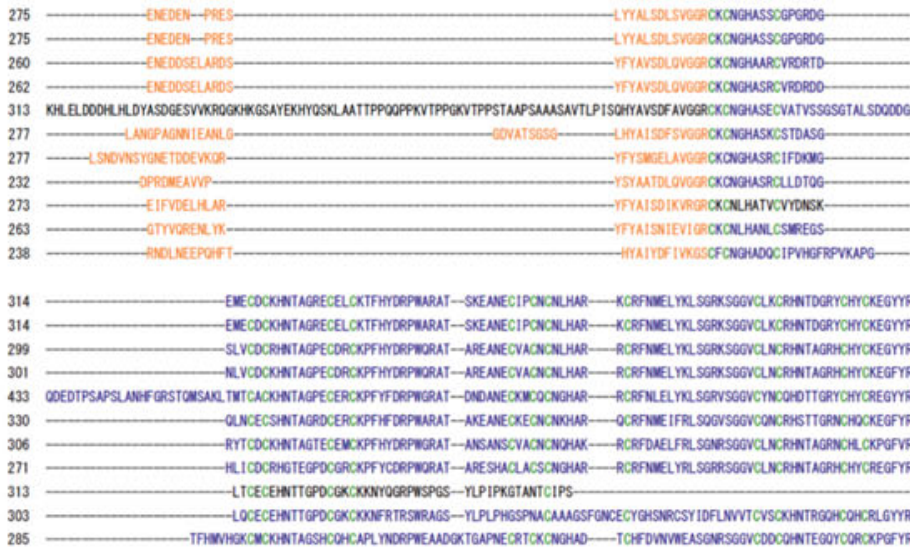

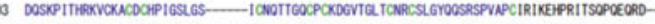

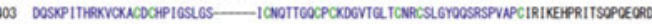

- MSSTEPPDC

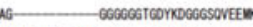

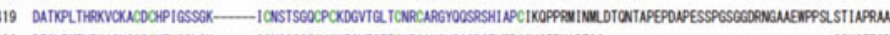

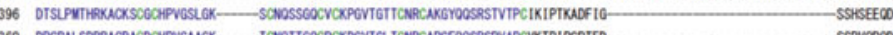

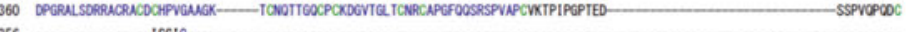

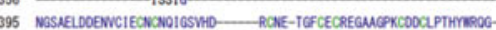

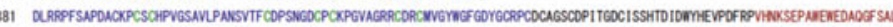

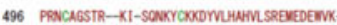

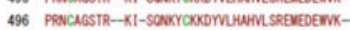

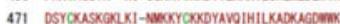

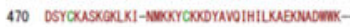

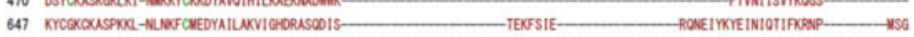

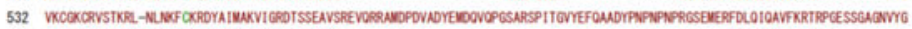

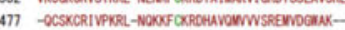

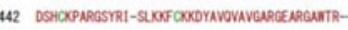

363 - TNCONELHCONGG

$451 \longrightarrow$ CrPworoonouconios

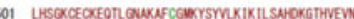

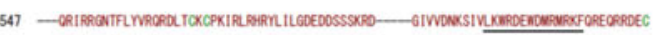

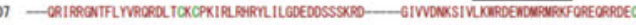

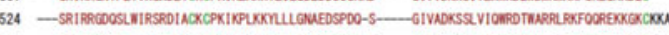

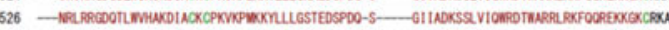

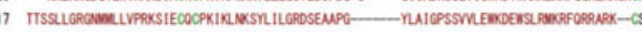

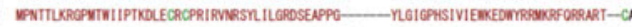

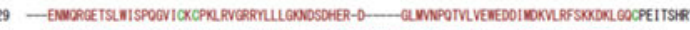

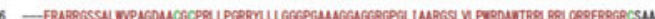

(9)

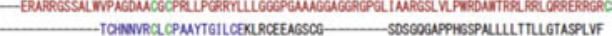
-TOANVRCL CP MYTTILCERLRCEEAGSCG

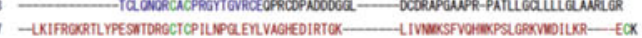

KIWESWFNGT-

623

623

604
604

al., 2000; Yaguchi and Katow, 2003), and the serotonin receptor cell network in the blastocoel (Katow et al., 2004; Katow et al., 2007). Like most neural wiring mechanisms, the neural plexus formation at the apical ganglion of the echinolarvae is associated with intricate weaving of the axons on the basal surface of the oral lobe ectoderm (Yaguchi et al., 2000). Initially the axons are extended from serotonergic cells that appear both on the left and right sides of the animal plate ectoderm toward the middle-ridge, where tall ectoderm cells comprise a ridge at the middle of the area, suggesting the presence of a chemoattractant that guides serotonergic axons to the middle-ridge. Such directional extension of the axons, particularly during central nervous system development in vertebrate embryos and nerve ring formation in $C$. elegans has been referred to as a "pathfinding" movement (e.g. Stoeckli and Landmesser, 1998; Zallen et al., 1999). The pathfinding mechanism involves such molecules as netrins, a group of extracellular matrix proteins (e.g. Serafini et al., 1994; Kennedy et al., 1994; Müller et al., 1996; Manitt et al., 2001; Tsai et al., 2003; Mann et al., 2004) along with three other sets of diffusible axonal chemoattractants described to date: Scatter factor/Hepatocyte growth factor (SF/HGF), Neurotrophin families, and a group of morphogens consisting of Shh (Charron et al., 2003), BMP (Augsburger et al., 1999) and Wnt family members (Hall et al., 2000; Wang et al., 2002).

Netrins were initially proposed as a midline-derived chemoattractants in vertebrate spinal cord (for review, Tessier-Lavigne and Goodman, 1996), and are a small family of highly conserved diffusible axon guidance cues. They have been extensively studied in flies, nematodes and mice (e.g. Chisholm and TessierLavigne, 1999). Netrins function in attraction or repulsion, depending on the type of their receptors (Manitt and Kennedy, 2002; Geisbrecht et al., 2003; Round and Stein, 2007). An attractive response functions with Deleted in Colorectal Carcinoma (DCC) (e.g. de la Torre et al., 1997; Deiner et al., 1997; Schwarting et al., 2004) whereas a repulsive effect occurs with UNC-5 (e.g. Leonardo et al., 1997; Keleman and Dickenson, 2001; Lim and Wadsworth, 2002; Guan and Condic, 2003).

Fig. 1. Amino acid sequence alignment of netrin proteins. Alignment of the predicted amino acid sequence of HpNetrin (Hp) compared to that of other reported netrin sequences in the $\mathrm{NBCl}$ database. Amino acids that comprise the VI domains are colored with orange, those comprising $V$ domain are with blue, and those comprising $C$ domain are with brown. Conserved cysteine residues are shown with green letters. $\mathrm{Sp}$, Strongylocentrotus pulcherrimus netrin (XP_001177385); $\mathrm{Hn}$ 1, human netrin-1 (NP_004813); Ggn-1, Gallus gallus netrin-1 (NP_990750); Dmn-B, Drosophila melanogaster netrin-B (NP_511155); Dmn-A, Drosophila melanogaster netrin-A (AAB 17533); Unc-6, Caenorhabditis elegans UNC-6 (AAA80449); Hn-3, human netrin 3 or netrin-2 like (NP_006172); HnG1, human netrin G1 (NP_055732); HnG2, human netrin G2 (NP_115925); Hn-4, human netrin-4 (NP_067052). The amino acid sequence used for raising antibodies is underlined. 
Based on these previous observations, it has been predicted that netrin-like chemoattractants function during serotonergic nervous system formation in sea urchin larvae. Netrin has been sequenced in sea urchins, such as Hemicentrotus pulcherrimus in 2005 (BAD91310) and Strongylocentrotus purpuratus in 2006 (XP_001177385). Other than sequences of the gene and the protein, no further characterization has been reported to date. The present study, thus, aimed to characterize the netrin-like protein, and to analyze its expression pattern in relation to serotonergic axon growth in sea urchin larvae. No other netrin-like protein has been reported to date in echinoderms.

In this study, I have characterized the hemichordate netrin-1 ortholog in the sea urchin, H. pulcherrimus (HpNetrin), and elucidated its structure, expression patterns of the gene and the protein as well as examined the role of HpNetrin in serotonergic axon guidance by cell culture in vitro and morpholino anti-sense oligonucleotide microinjection.

\section{Results}

\section{Structure of the netrin homolog in $\mathrm{H}$. pulcherrimus and its transcription pattern during early development}

The open reading frame of the cDNA was comprised of 1,869 bps that resided between a 551 bp 5' UTR and 1,061 bp 3' UTR regions. It encoded 623 amino acids. Protein alignments among netrin homologs showed the well-conserved domain structures, except that netrin $\mathrm{A}$ and $\mathrm{B}$ of $D$. melanogasterand human netrin G1 and G2 lack the $C$ domain (Fig. 1). Likewise, the cysteine residues were well conserved among the netrin homologs and the $\mathrm{VI}, \mathrm{V}$ and $\mathrm{C}$ domains of $\mathcal{S}$. purpuratus netrin were practically identical to these of netrin of $H$. pulcherrimus. A phylogenetic tree constructed based on the full-length amino acid sequences among netrins and human laminin referred in this study indicated that netrin of $H$. pulcherrimus (HpNetrin) belongs to a group of deuterostomes that includes netrins of amphioxus, hemichordate, ascidian, and netrin-1s of human and avian, and quite distant from other two groups of protostomes, and human netrin-4 (Fig. 2). HpNetrin was similar to netrin-1 of hemichordate, Saccoglossus kowalevskii with bootstrap value $73.3 \%$. (Fig. 2). Thus, although the bootstrap value was a little too low, the present netrin of $H$. pulcherrimus could be an ortholog of hemichordate netrin-1. According to protein sequences, two $\mathcal{S}$. purpuratus netrins (for full-length amino acids sequence: XP_001177385 and $\left.X P \_781902\right)$ are identical, and only two amino acids were different from the 623-amino acid HpNetrin.

According to RT-PCR, the Hpnetrin transcript was detected from 15-hour post-fertilization (15-hpf) swimming blastula stage to 48-hpf pluteus stage with apparently high activity from 16-hpf mesenchyme blastula stage to 24 -hpf prism stage (Fig. 3). No transcript accumulation was seen before the 15-hpf swimming blastula stage. Although the onset of transcription was well before the apical ganglion formation at 24-hpf prism stage (e.g. Yaguchi et al., 2000; Yaguchi and Katow, 2003), it distinctively decreased in 48-hpf plutei when formation of the serotonergic nervous system was still in progress.

\section{Protein expression pattern during development}

Immunoblotting using a sample from 18-hpf early gastrulae showed the antibodies (Ab) bound exclusively to a band at $75 \mathrm{kDa}$ region (Fig. 4, lane 1) that was close to known netrins sizes (Chisholm and Tessier-Lavigne, 1999; Koch et al., 2000). The Ab binding to the band was blocked considerably in the presence of $1 \mathrm{mg} / \mathrm{ml}$ synthetic peptide used for raising the Ab (Fig. 4, lane 2), indicating the Ab specifically bound to a band that contains the amino acid sequence used for antigen peptide. The relative

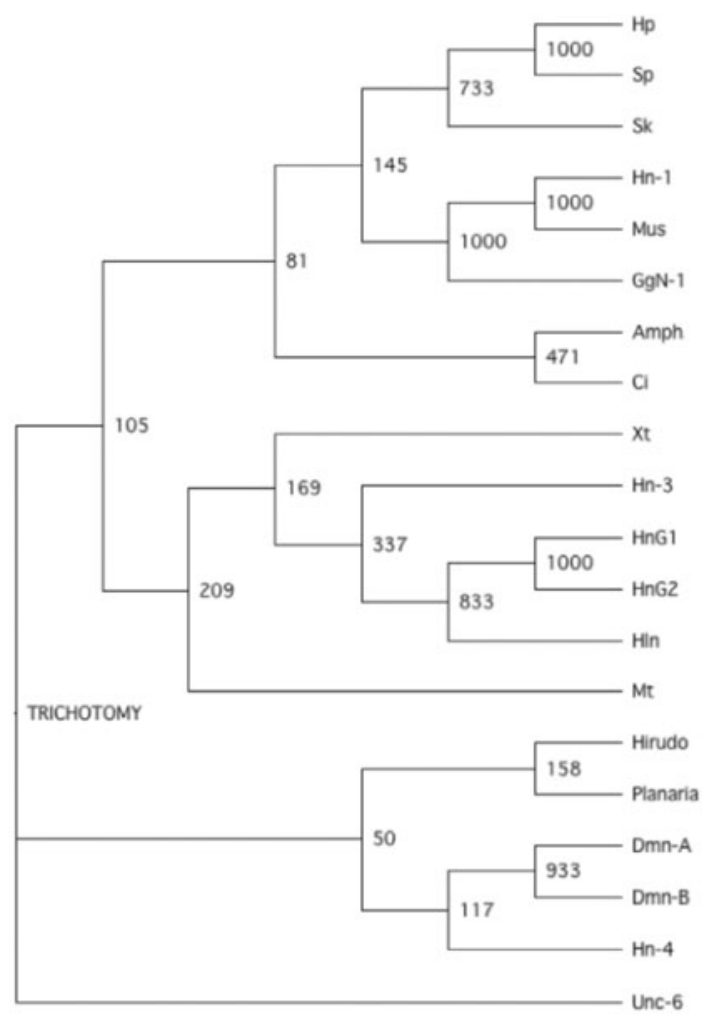

Fig. 2. Neighbor joining tree based on full-length amino acid sequences of netrins. Numbers indicate bootstrap values 1,000 replicates). In addition to the netrins aligned in Fig. 1, the following 8 netrins were analyzed. Amph, amphinetrin of Branchiostoma floridae (CAB72422); $\mathrm{Ci}$, netrin of Ciona intestinalis (NP_001027598); Hirudo, netrin precursor of Hirudo medicinalis (AAC83376); HIn, human laminin (NP_002284); Mt, netrin of Molgula tectiformis (BAF35976); Mus, netrin 1 of Mus musculus (NP_032770); Planaria, netrin-1 of Schmidtea mediterranea (AAY23350); Sk, netrin-1 of Saccoglossus kowalevskii ABD97277; Xt, netrin-1 of Xenopus tropicalis (NP_001072385). Bar shows evolutionary distance.

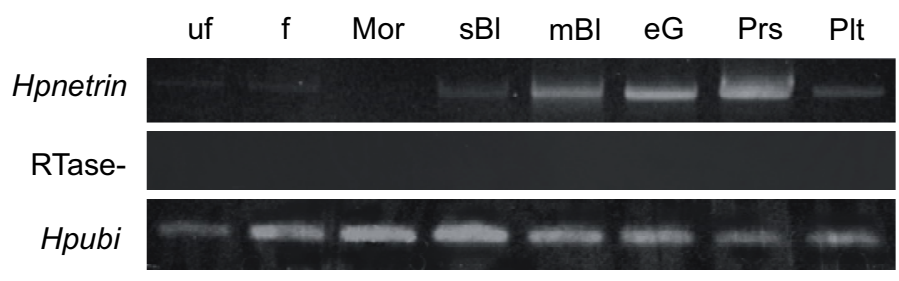

Fig. 3. Reverse transcriptase-PCR analysis of Hpnetrin transcription. The transcription of Hpnetrin occurs from 15-hpf swimming blastula stage (sBl) to 48-hpf pluteus stage (Plt). Uf, unfertilized eggs; f, eggs 30 min after fertilization; Mor, 5-hpf morulae; mBI, 16-hpf mesenchyme blastulae; eG, 18-hpf early gastrulae; Prs, 24-hpf prism larvae. RTase-, without reverse transcriptase; Hpubi, with Hpubiquitin primers. 


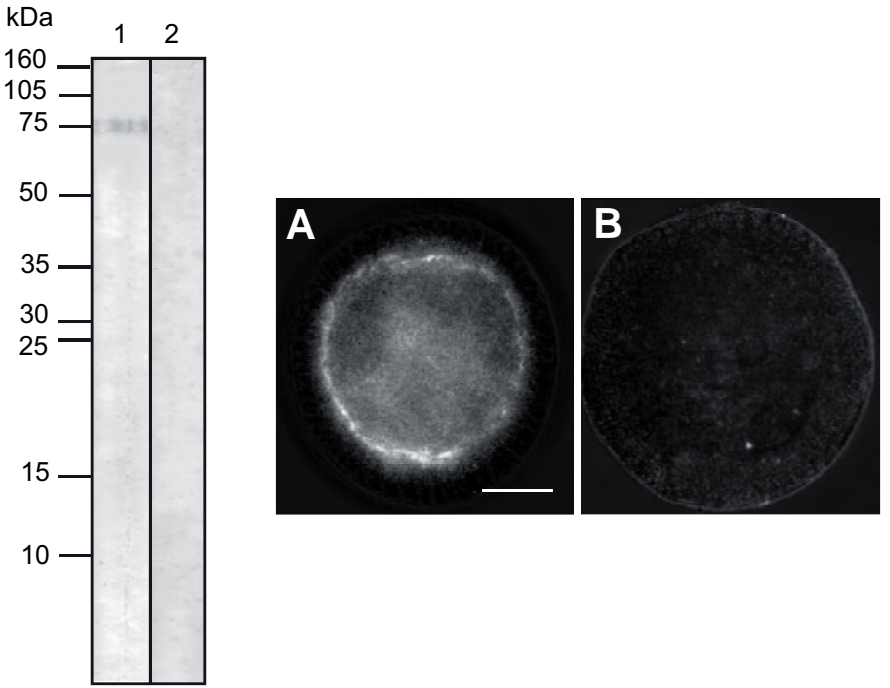

Fig. 4. Immunospecificity of anti-HpNetrin antibodies. According to immunoblotting, the antibody detects a band in the $75 \mathrm{kDa}$ region (lane 1) that is significantly inhibited in the presence of $1 \mathrm{mg} / \mathrm{ml} \mathrm{HpNetrin}$ peptide (lane 2) used for raising the antiserum. Whole mount immunohistochemistry of the antibody localizes HpNetrin on the basal surface of 16 hpf mesenchyme blastula (A). This immunoreactivity is inhibited in the presence of $3 \mathrm{mg} / \mathrm{ml}$ synthetic peptide (B), indicating immunospecificity of the antibody. Scale bar, $40 \mu \mathrm{m}$.

molecular mass of the band was close to that calculated from 632 -amino acid protein $(71 \mathrm{kDa})$ according to Protein Identification and Analysis Tools on the ExPASy Server based on UniProt Knowledgebase (Swiss-Prot or TrEMBL) (http:// us.expasy.org/tools/pi_tool.html).

Immunospecificity of the $A b$ in whole mount immunohistochemistry (WMIHC) was examined with or without the synthetic peptide used for raising $\mathrm{Ab}$. The $\mathrm{Ab}$ bound to the entire basal surface of mesenchyme blastula without the peptide (Fig. $4 \mathrm{~A}$ ) and was considerably decreased with $3 \mathrm{mg} / \mathrm{ml}$ of the peptide (Fig. 4B), indicating the Ab specifically recognized the protein on the basal surface of embryonic ectoderm. Thus, the present $A b$ recognizes Hpnetrin selectively.

Immunoblotting showed that Hpnetrin expression started around the 15-hpf swimming blastula stage (Fig. 5, lane 4). Intensity of the immunoreaction remained similarly toward 48hpf pluteus stage (Fig. 5, lane 9). No visible immunoreaction was seen in unfertilized eggs, in eggs 30 minutes after fertilization, and in morulae. The present observation of immunoblotting was consistent with the above RT-PCR analysis (Fig. 3) and with the following WMIHC (Fig. $5 \mathrm{~A}-\mathrm{G}$ ), suggesting that $\mathrm{HpNetrin}$ expression was zygotic.

According to the present WMIHC, HpNetrin was localized on the entire basal surface of 15-hpf swimming blastulae and 16-hpf mesenchyme blastulae (Fig. 5 A,B). This, however, was changed at and after 18-hpf early gastrula stage. The immunoreaction in 18-hpf mid-gastrulae increased a little at the animal plate, while in the rest of the ectodermal regions it decreased (Fig. 5C). In 24-hpf prism larvae, HpNetrin distribution showed considerable heterogeneity that was comprised of three regions. 1) It was prominent by the lateral view of the larva. The most posterior aboral ectoderm showed the highest intensity of HpNetrin immunoreaction (area-I), 2) the most anterior oral ectoderm showed the lowest intensity (area-III), and 3) a narrow ectodermal region in between these two regions showed moderate intensity (area-II, Figs. 5D, 6 E-I, 7A). The oral side view of 24-hpf prism showed a patch of distinctive $\mathrm{HpNetrin-rich} \mathrm{area} \mathrm{at} \mathrm{the} \mathrm{animal} \mathrm{plate} \mathrm{that} \mathrm{gradually}$ decreased toward both left and right adjacent ectodermal regions (Fig. 5E). The oral-aboral polarity of HpNetrin distribution was more prominent in 48-hpf plutei. The immunoreaction of HpNetrin intensified at area-I (Fig. 5F), while area-III remained with weaker immunoreaction and area-II also remained moderate. Ventral view of the larva showed a distinctive HpNetrin positive thin strip between two arms that became conspicuous by 48 -hpf pluteus stage (Fig. 5G, arrow) that comprises the ciliary band.

The double immunostaining localized an initial serotonergic cell that extended short axon to the middle of the animal plate
Fig. 5. Time course of HpNetrin expression during early development. Immunoblotting shows that HpNetrin is not expressed in unfertilized eggs (lane 1), in eggs 30 min after fertilization (lane 2), or in morulae (lane 3). In contrast, the protein is expressed distinctively from 15-hpf swimming blastulae (lane 4) to, at least, 48-hpf plutei (lane 9). Lane 5, 16-hpf mesenchyme blastulae; lane 6, 18-hpf early gastrulae; lane 7, 20-hpf mid-gastrulae; lane 8, 24-hpf prism larvae. Confocal laser scanning micrographs of whole mount immunohistochemical preparations indicates that HpNetrin is expressed on the entire basal surface in swimming blastula (A) and in mesenchyme blastula (B). Overall immunoreaction slightly decreases in intensity around the vegetal pole from the 18-hpf mid-gastrula stage (C) and distinctively in 24-hpf prism larva stage (D,E). Although the oral view shows distinctive immunoreaction around the animal plate (E), the left side view shows strong immunoreaction on the aboral side and weak on the oral side (D, asterisk). At 48-hpf pluteus stage $(\mathbf{F}, \mathbf{G})$ HpNetrin is expressed more prominently than in prism larvae on the aboral ectoderm side (F), while it decreases on the oral side (asterisks). HpNetrin also is seen between 2 arms around the ciliary band (G, arrow). Scale bar, $20 \mu \mathrm{m}$.

\begin{tabular}{|lllllllll|}
1 & 2 & 3 & 4 & 5 & 6 & 7 & 8 & 9 \\
\hline & & & & & & & & \\
\hline
\end{tabular}
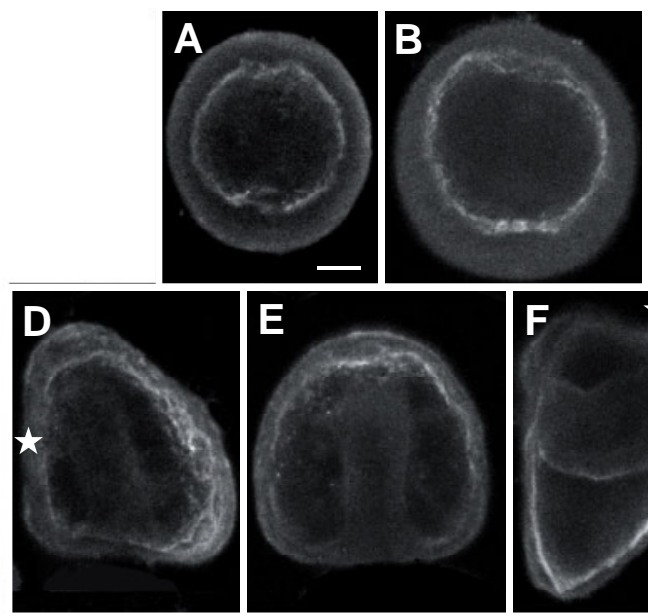

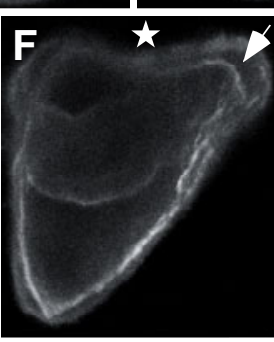

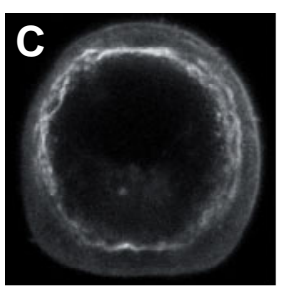

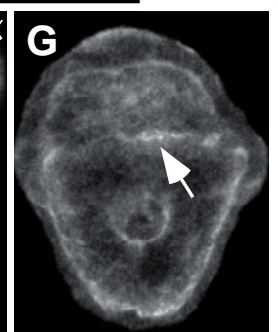


region at the periphery of $\mathrm{HpNetrin-positive} \mathrm{animal} \mathrm{plate} \mathrm{in} \mathrm{27-}$ hpf prism larvae (Fig. 6A). An increase of serotonergic cell numbers was seen by 30 -hpf prism stage. A row of these cells was aligned at right angle to the larval antero-posterior or oralaboral axis at HpNetrin-positive animal plate. The oral side view showed that their apico-basal axis did not reach to the apical surface of the ectoderm, suggesting they were somewhat tilted along the oral-aboral axis (Fig. 6B). The cell alignment, however, appeared along the oral-aboral axis that was initiated by an oral most serotonergic cell at the periphery of HpNetrinpositive ectoderm (Fig. 6C, arrow). According to section-tosection analysis of this stacked image, these serotonergic cells were in fact separated from the $\mathrm{HpNetrin-rich} \mathrm{animal} \mathrm{plate} \mathrm{area}$ at about $3 \mu \mathrm{m}$, and aligned around the periphery of the area. These serotonergic apical ganglion cells started to extend their axons by 41-hpf prism stage. The oral side view showed that these cells extend their axons toward the middle of HpNetrinrich animal plate (Fig.6D, arrow). The side view of the larva, however, showed that these axons were not exactly extended toward the middle of $\mathrm{HpNetrin-rich} \mathrm{animal} \mathrm{plate} \mathrm{region} \mathrm{with} \mathrm{right}$ angle to the anterior-posterior axis of the larva, but toward
HpNetrin-rich posterior ectodermal region (Fig. 6E, arrow, 7A) Furthermore, these serotonergic cells, again, did not sit in an $\mathrm{HpNetrin-rich} \mathrm{ectodermal} \mathrm{region,} \mathrm{but} \mathrm{at} \mathrm{the} \mathrm{periphery} \mathrm{of} \mathrm{the}$ region in area-II (Figs. 6E, inset, 7A). The serotonergic axons extended further to form a neural plexus on the basal side of serotonergic cells by 48-hpf pluteus stage (Fig. 6F). By 53-hpf pluteus stage, most of these axons from apical ganglion extended further laterally to the left and right lateral ectodermal regions of the oral lobe (Fig. 6G). However, axons also extended posteriorly from the middle of the apical ganglion (Fig. $6 \mathrm{H}, \mathrm{I})$. These axons extended on the basal surface of the dorsal ectoderm, which was rich in HpNetrin, and formed an approximately $40 \mu \mathrm{m}$ wide belt from the immediate posterior edge of area-II to near the posterior end of the larval body (dorsal midline belt, DMB) by $41 \mathrm{~h}$-phf pluteus stage (Figs. 6E, 7). The middle-ridge was no more a distinctive feature of the animal plate at this developmental stage (Fig. 6F). According to the present confocal laser scanning microscopy, initial DMB was seen from around 30-hpf prism stage (Figs. 6C, 7 A,B), and morphologically more distinctive from 41-hpf to 48-hpf pluteus stage (Figs. 6E, 7 C,D).
Fig. 6. Confocal laser scanning micrographs and fluorescence micrographs of double-stained whole mount immunohistochemistry preparations. Serotonergic cells were stained with green and HpNetrin with red. (A) 27-hpf prism. Serotonergic cells appeared at the periphery of the HpNetrin-positive animal plate. (B) Oral view of 30-hpf prism. Serotonergic cells aligned apparently at right angles to the oral-aboral axis of the larva at HpNetrinpositive animal plate. (C) Left side view of 33-hpf prism. HpNetrin expressing region of the ectoderm was seen from the animal plate region to a little closer to the vegetal pole on the aboral ectoderm, while the rest of the ectodermal regions, including oral ectoderm show little or no HpNetrin. Three serotonergic cells locate to the HpNetrin-positive animal plate region. (D) Oral view of 41-hpf prism. The serotonergic cell on the left hand side extends axons toward the middle of the HpNetrin-expressing animal plate (arrow). (E) Right side view of 41-hpf prism. Serotonergic cells reside in the place where HpNetrin is expressed moderately, followed posteriorly by the protein-intensive ectoderm (also see inset). An axon from a serotonergic cell is extended to HpNetrin-rich ectoderm (arrow). This $11 \mu$ m-thick image was stacked to include serotonergic cells, broadened HpNetrin-rich dorsal ectoderm, including a space between digestive organs and the dorsal ectoderm. Inset shows a thinner ( $3 \mu \mathrm{m}$ thick) stacked image of the same region. The dorsal ectoderm is separated with 1 to $6 \mu \mathrm{m}$ wide space from the dorsal side of the digestive organs (e, esophagus, s, stomach). (F) Dorsal view of 48-hpf pluteus. Serotonergic axons formed a neural plexus between two clusters of serotonergic cells. (G) Dorsal view of 53-hpf pluteus. This particular larva extends serotonergic axons from the apical ganglion toward the left side ectoderm of the oral lobe. A $40 \mu \mathrm{m}$ wide HpNetrin-rich dorsal belt is clearly seen under the dorsal ectoderm (double-headed arrow, DMB). (H) Dorsal view of 48-hpf pluteus. Two serotonergic axons extended posteriorly on the DMB (doubleheaded arrow). (I) Dorsal view of 53-hpf pluteus. A serotonergic axon posteriorly extended from the middle of the apical ganglion of the DMB. (J) Immunofluorescence micrograph of an apical ganglion area of 58-hpf pluteus larva squashed a little under the
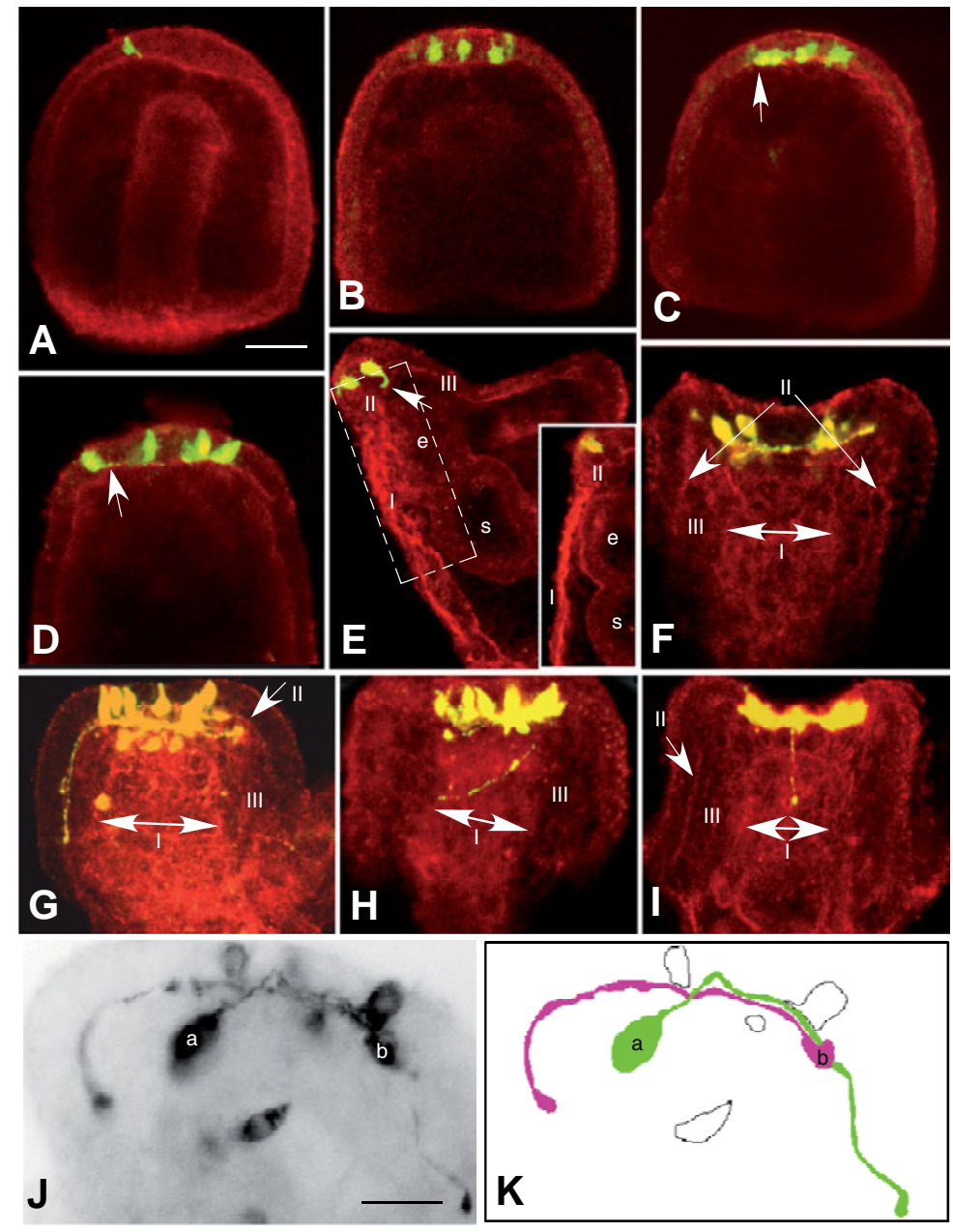

cover slip to disentangle the neural plexus to reveal individual

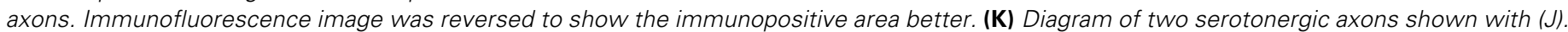

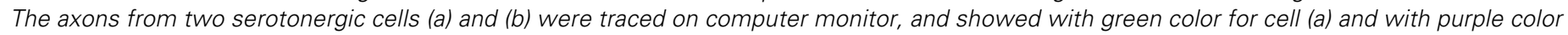
for cell (b). I, II and III in (E) to (I) show HpNetrin-rich area I, -moderate area II, and-poor area III, respectively. Scale bars in (A, J), 20 Hm. 
A
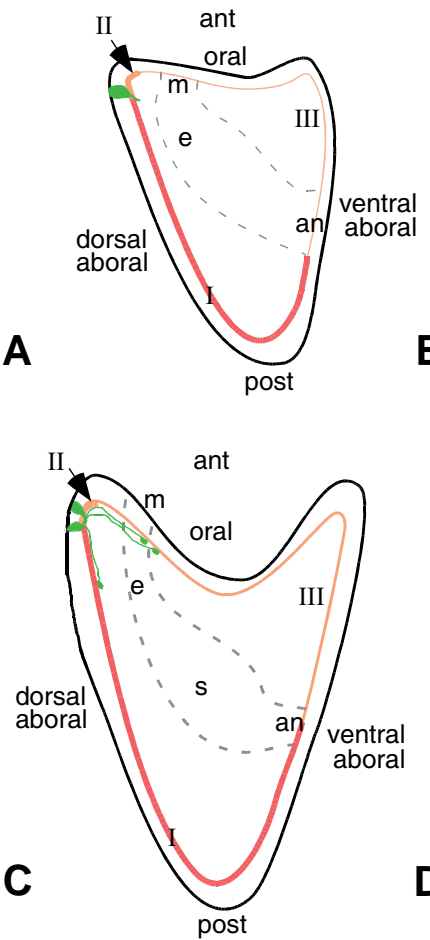

B
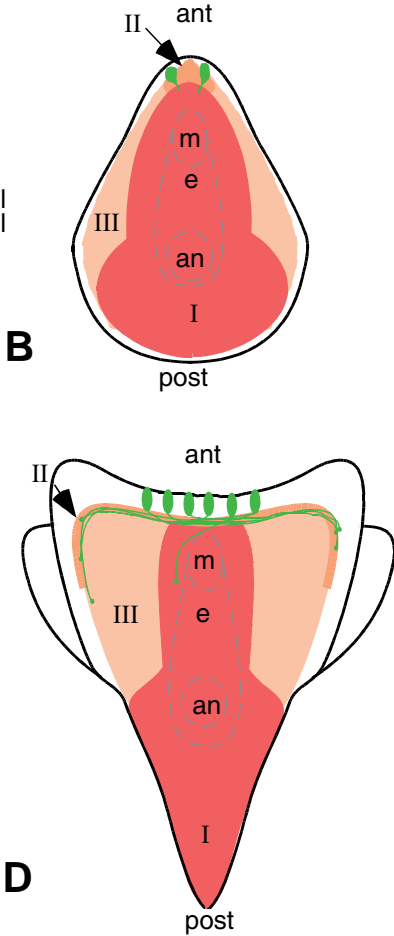

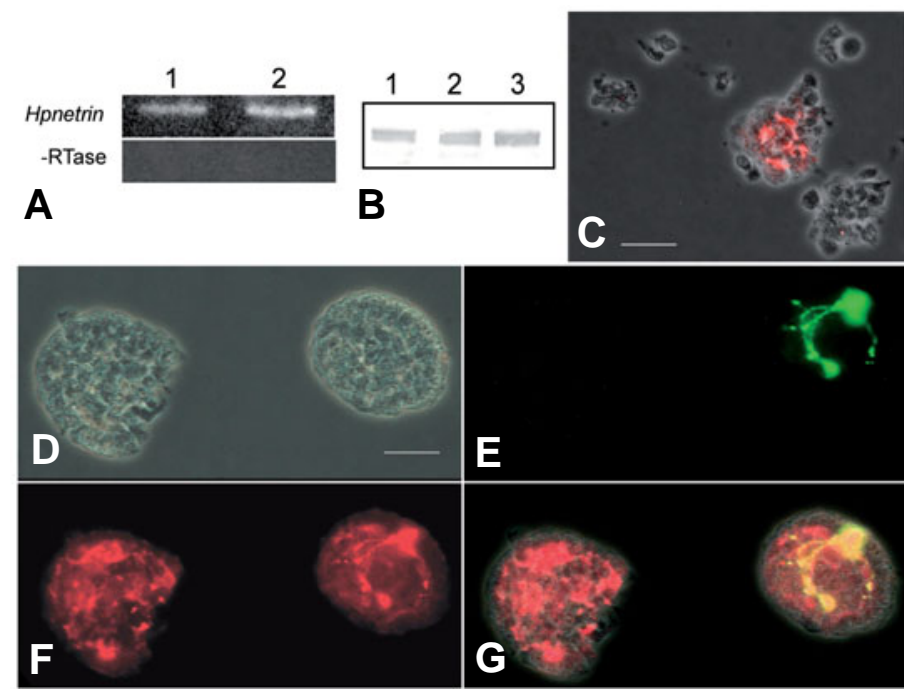

Fig. 7 (Left). Diagram of the spatial distribution of HpNetrin and extension pattern of serotonergic axons during larval development. (A) Right side view of prism. HpNetrin is rich in area-l (red color) that comprises most of the dorsal aboral ectoderm and the posterior ventroaboral ectoderm (from immediately posterior position of area-ll to the posterior side around the anus (an)), scarce in area-III (flesh color) that comprises most of the oral ectoderm and the antero-ventral aboral ectoderm (from immediately anterior side to area-II to the anterior side of

the anus), and moderate in area-Il (orange color) that locates in between and includes serotonergic cells. Serotonergic axons extend posteriorly toward HpNetrin-rich area-I. (B) Dorsal view of prism. Serotonergic axons extend posteriorly toward HpNetrin-rich area-I that comprises a dorsal midline belt (DMB). (C) Right side view of early pluteus. HpNetrin distribution pattern remains the same as in the prism. Serotonergic axon extension takes two routs; most of the axons extend ventrally toward area-III and some of them posteriorly on DMB. (D) Dorsal side view of early pluteus. Serotonergic axons extend on the area-ll ectoderm toward the contralateral ectoderm of the oral lobe after forming a neural plexus around the anterior area-I ectoderm. DMB is shown as a $40 \mu \mathrm{m}$ wide belt. ant, anterior; e, esophagus; $m$, mouth; s, stomach; post, posterior. Dotted lines show outline of digestive organs.

Fig. 8 (Right). Hpnetrin transcription and the protein expression in mini-blastulae. (A) Hpnetrin transcription was not disturbed during dissociation of embryos (lane 1) and was retained in mini-blastulae after 24 hours of in vitro culture (lane 2). (B) Immunoblotting shows HpNetrin expression in intact embryos (lane 1), in embryonic cells soon after dissociation (lane 2) and in mini-blastulae after 24 hours of in vitro culture (lanes 3). (C) Immunofluorescence micrograph of mini-blastula that expressed HpNetrin, and that remained either as single cells or formed cell aggregates with smaller number of cells which did not express the protein. (D) Light field micrograph of two mini-blastulae formed 24 hours after artificial dissociation. (E) Immunofluorescence micrograph shows serotonergic cells differentiated with long axons in the right mini-blastula (green). (F) Immunofluorescence micrograph of the same mini-blastulae shows HpNetrin expression (red). (G) Immunofluorescence micrograph shows that HpNetrin expression is not always correlated with serotonergic cell differentiation as shown with this merged image. Serotonergic cell in the right side mini-blastula expresses HpNetrin (yellow). Scale bars in (C,D), $30 \mu \mathrm{m}$.

During neural plexus formation at the apical ganglion, axons from serotonergic cells extended toward the contralateral ectoderm of the oral lobe region. Fig. $6 \mathrm{~J}$ shows such axon extensions in the oral lobe after lightly squashing the larva to reveal single neurites. A serotonergic cell on the left (Fig. 6J, "a" cell) extended its axon toward the right side, while another cell on the right side (Fig. 6J, "b" cell) extended its axon toward the left side as diagramed with Fig. 6K. Thus, the neural plexus was made at the place where axons extended from both left and right sides of the HpNetrin-rich animal plate ectoderm beneath the serotonergic cells of the middle-ridge. After passing through the neural plexus, each axon extended away from the HpNetrinrich region that is a contrary response to that before reaching to the middle-ridge. This also was seen in axons that extended posteriorly from the middle of apical ganglion toward the DMB (Fig. 6I). They extended in the DMB some distance and then changed the direction away from the HpNetrin-rich region toward the lateral boundary of the DMB that adjacent to HpNetrinpoor ectodermal region (Fig. 6H). Thus, serotonergic axons extended initially toward HpNetrin-rich middle-ridge or DMB, and after crossing the middle-ridge or spending some period in DMB, the axons moved away from HpNetrin-rich region.

Such a reversal response of axons to $\mathrm{HpNetrin-rich} \mathrm{region,}$ however, occurred moderately. No axons reached the HpNetrindeficient ectodermal area, such as the apical surface of ectoderm. This implicates that the serotonergic axons retained their interactive relation with $\mathrm{HpNetrin}$ throughout their extension period regardless of an attractive or repulsive response. HpNetrin was also observed occasionally on the serotonergic cells and their axons in larvae older than 48-hpf plutei, and more often from 53-hpf pluteus stage (Fig.6 F-I). This location resembled netrin on nervous cells, such as chick cutaneous neurons (Guan and Condic, 2003) and implicates the continuous influence of HpNetrin throughout axon extension. Thus, the present 
observation suggests that HpNetrin is necessary as a serotonergic axon guidance cue.

\section{Co-localization of HpNetrin and serotonergic axons in an artificial micro-environment}

To examine whether HpNetrin influences extension of serotonergic axons even under an anatomically perturbed environment, 20 -hpf early gastrulae were artificially dissociated to single embryonic cells and incubated in vitro in conditions to promote cell-aggregates. In these 20-hpf gastrulae, Hpnetrin transcription (Figs. 3, 8A, lane 1) and protein expression (Figs. 5A, 8B, lanes 1 and 2) were already in progress but the serotonergic cells and HpNetrinrich animal plate region had not yet formed (Fig. 5B). After 24 hours of in vitro incubation, dissociated embryonic cells formed small cell-aggregates of $50 \mu \mathrm{m}$ in diameter on average (Figs. 8C,D, 9). According to RT-PCR, these embryonic cells actively transcribed Hpnetrin (Fig. 8A, lane 2). Immunoblotting showed that HpNetrin protein was also expressed in these cells (Fig. 8B, lane 3). This indicated that conservation of intact anatomical structure is not required for HpNetrin expression. However, when one closer look at the present immuno-positive banding pattern, unlike immunoblotting pattern seen using intact plutei (Fig. 4), this showed closely associated two bands at lanes 1 and 2, but not at lane 3 .

WMIHC showed that the embryonic cells that did not form cell-aggregates or even remained as single cells did not express HpNetrin (Fig. 8C), while those that formed small cell-aggregates developed a cavity in the middle that was lined with a single cell-layer (Figs. 8C,E, 9). The single cell-layer expressed HpNetrin exclusively on the side of cavity (basal surface of the cavity, Figs.8E, 9) and cilia on the outer surface (apical surface), indicating that the apicobasal polarity of ectoderm is retained in these cell-aggregates. This resembled the blastula morphology (miniblastula) (Fig.5 A, B). In many of these mini-blastulae, a few serotonergic cells were formed with axons extended on the basal surface of the cavity, particularly to the places where HpNetrin is expressed distinctively (Figs. 8F, 9). A striking property of these axons was that none of them were extended to the outside of the mini-blastulae, where HpNetrin was not expressed. Instead, the axon location was restricted to within the $\mathrm{HpNetrin-expressing} \mathrm{region} \mathrm{(Figs.} \mathrm{8G,}$ 9D). Furthermore, these serotonergic cells themselves occasionally expressed HpNetrin on their surface (Figs. 8G, $9 B$ ), as was seen in axons of the intact larvae (Fig.6 F-I).

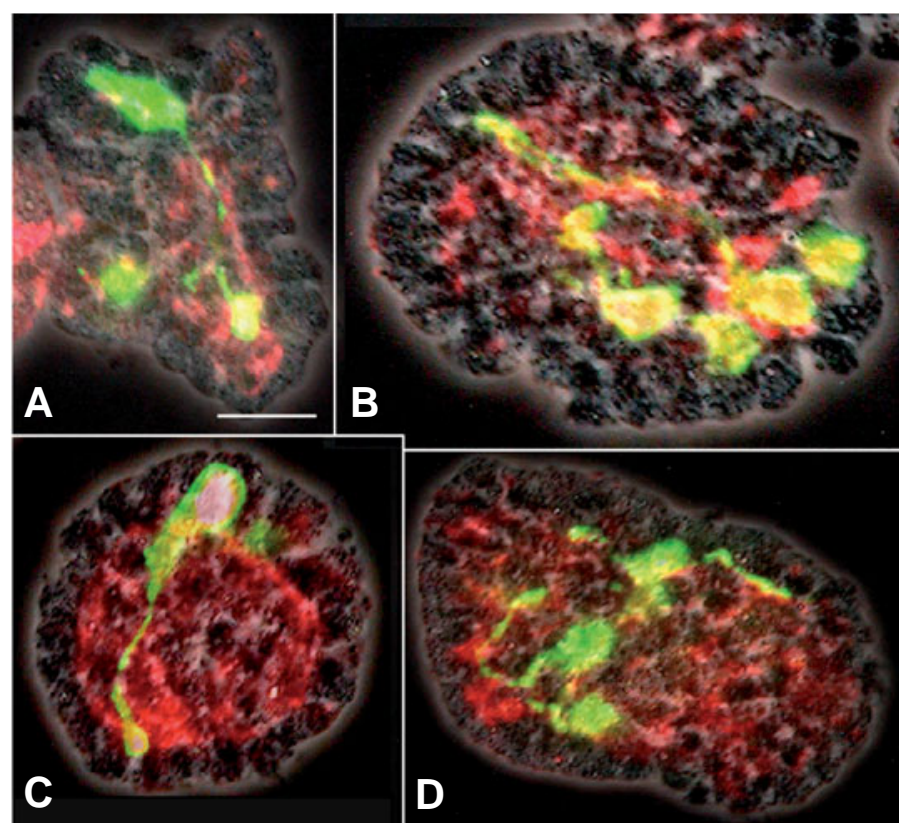

Fig. 9. Immunofluorescence micrographs of serotonergic axon extension patterns in mini-blastulae. Serotonergic axons (green) never extend to the outer surface of mini-blastulae. (A) A serotonergic axon extends on HpNetrin-positive area (red). (B) Four serotonergic cells were formed in this mini-blastula. All of them express HpNetrin (yellow). A serotonergic axon at the second from the right extends through HpNetrin-positive area. (C) $A$ serotonergic cell extends its axon toward a HpNetrin-rich area cross the cavity of mini-blastula. (D) Two serotonergic cells extend their bipolar axons on HpNetrin-positive areas. Scale bar in (A), $20 \mu \mathrm{m}$.

These present observations implicate that HpNetrin expressed on the basal surface of the ectoderm functions attracted serotonergic axons.

\section{Loss-of-function analysis using morpholino anti-sense oligonucleotide (MASO)}

In 56-hpf plutei that were microinjected with either $10 \mu \mathrm{M}$ or $20 \mu \mathrm{M}$ of MASO resulted in either no effect or severe inhibition of morphogenesis (data not shown), whereas $12 \mu \mathrm{M}$ of MASO showed selective inhibition. In these larvae, overall morphology of pluteus was retained, and serotonergic cells were differentiated, but their axon extension was severely inhibited to show a cluster of serotonergic cell bodies at the apical ganglion area (Fig.10A). HpNetrin distribution also was severely perturbed so that area-II was virtually not formed at the apical ganglion area. Whereas, in

Fig. 10. Confocal laser scanning micrographs of double stained immunohistochemistry of Hpnetrin MASO-injected 56-hpf larvae. (A) Serotonergic cells (yellow) failed to extend their axons in Hpnetrin MASO-injected larva, and HpNetrin distribution (red) was severely perturbed so that area-ll was not recognized beneath the serotonergic ganglion. (B) In larva that was injected with standard control oligonucleotide, serotonergic axons extend to both
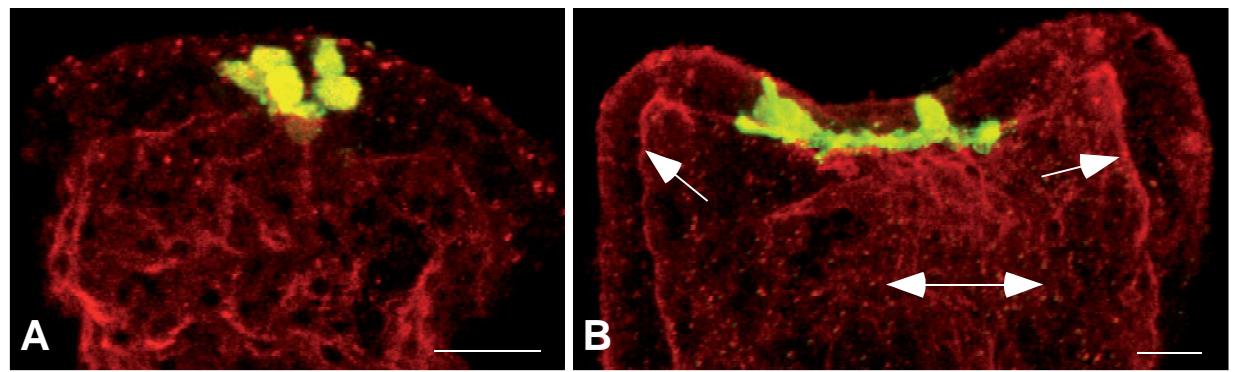
left and right side of the ganglion. HpNetrin formed clear area-Il (arrows) and dorsal midline belt (double headed arrow).Scale bars in (A,B), 20 um. 
plutei that were injected with standard control oligonucleotide, none of these abnormalities were recognized (Fig.10B).

\section{Discussion}

During the early prism stage, serotonergic cells formed around the animal plate. They extended their axons in the space between the basal lamina and the ectoderm initially toward the middle-ridge (Yaguchi et al., 2000), and suggesting the presence of axon guidance cues probably provided by the middle-ridge region. This region is comprised of characteristically tall cells that form a thickened ectoderm area in the middle of the animal plate. Such directional axon extension towards the middle-ridge or DMB resembled the axon guidance at the ventral midline in nematodes, fruit flies, and vertebrates (for review, Tessier-Lavigne and Goodman, 1996). The midline tissues of these animals are derived from the ectoderm, as in the middle-ridge and DMB of sea urchin larvae. However, unlike midline tissue, such as of Drosophila (Bossing and Brand, 2006), the DMB does not produce any known nerve cells. Apparently the midline and the DMB are not comparable tissues. CNS midline cells are specified by the single-minded (sim) gene in Drosophila (Lee et al., 1999), and 224 genes expressed in the midline were identified (Kearney et al., 2004; Wheeler et al., 2006), but not yet in DMB of the sea urchin larvae. Only a few of them, including 5-HThpr(a serotonin receptor gene) (Katow et al., 2004) and zfh1 (Burke et al., 2006) have been identified in the sea urchin. However, the serotonin receptor of the sea urchin is not expressed in the DMB (Katow et al., 2004). Sim is not yet identified in sea urchin, and $z$ fh 1 expression site is not yet known.

\section{Protein property and phylogenetic position of HpNetrin}

The open reading frame of Hpnetrin encoded 623 amino acids that constituted 3 major domains, $\mathrm{VI}, \mathrm{V}$ and $\mathrm{C}$ domains from the $\mathrm{N}$ terminus end, and its relative molecular mass $(75 \mathrm{kDa})$ fitted in the range of known netrins ( 70-80kDa, Chisholm and Tessier-Lavigne, 1999). The present phylogenetic tree showed that among netrins referred in this study, sea urchin netrins are ortholog of netrin-1 of hemichordate, and well fit in the classic phylogenetic tree. Almost identical amino acid sequences between $\mathrm{HpNetrin}$ and $S$. purpuratus netrin provided additional support that these sea urchins are sister species. Two netrins of $\mathcal{S}$. purpuratus registered to NCBI (XP_001177385 and XP_781902) are in fact not distinctive netrins. Their 623-amino acid sequences are nearly identical with only two amino acids different between them. Thus, sea urchins appear to contain a single netrin protein.

\section{Spatio-temporal protein expression pattern during serotoner- gic nervous system formation}

Transcription of Hpnetrin occurred initially at the 15-hpf swimming blastula stage and remained active until the prism stage. Toward the pluteus stage, the gene transcription decreased considerably. However, serotonergic cells appear from the prism stage toward the pluteus stage, extending axons to form a neural plexus beneath the serotonergic apical ganglion and then constructing the remainder of the serotonergic nervous system (e.g. Yaguchi et al., 2000). This histological observation based on WMIHC is supported by Northern blotting analysis of the transcription period of the tryptophan 5-hydroxylase gene of $H$. pulcherrimus
( HpTPH) , a serotonin synthetase. Northern blotting detects HpTPH transcription from prism stage to pluteus stage (Yaguchi and Katow, 2003). Since active serotonergic axon extension and serotonergic nervous system construction occur at and after the prism stage, HpNetrin appears to be not involved in serotonergic axon guidance cue. However, this apparent lack of linkage between activities of these two genes during the formation of serotonergic nervous system is superficial. HpNetrin protein expression was sustained, even after the prism stage, until the pluteus stage based on both immunoblotting and WMIHC shown here, indicating that despite decreased Hpnetrin gene transcriptional activity, its product is maintained in the developmental period. The nervous system expansion continues after 48-hpf pluteus stage, despite of considerable decreasing of Hpnetringene transcription (Fig. 5A). Although molecular life span of HpNetrin is not yet clear, it is possible that the other axon guidance cues, such as semaphorins and/or ephrins, replace HpNetrin after decreasing of the protein production. In fact, semaphorin-1 (accession number XM_001187939) and ephrin-2, as is suggested by the presence of its receptor (accession number XM_001186578), of Strongylocentrotus purpuratusare cloned, and registered to NCBI. However, further examination other than the sequences of DNA and protein is yet to come.

WMIHC results herein show that HpNetrin is expressed on the basal surface of the ectoderm from the prism stage with a dynamic spatial distribution pattern change afterward during serotonergic nervous system formation. In 53-hpf plutei, the HpNetrin expressing area expanded to the posterior ectodermal region with intensive immunoreaction at the ciliary band (Fig. 5G). Serotonergic axons do not yet reach to this far posterior region, but antisynaptotagmin Ab-positive nervous cells (SPC) form a nervous network under the ciliary band ectoderm and the nervous network stretches toward the posterior ectoderm (Nakajima et al., 2004). Thus, HpNetrin could also be involved in this SPC network formation

Curiously, HpNetrin is expressed well before the onset of serotonergic axon extension, such as in mesenchyme blastulae (Fig. 5B). Recent advancement in our understanding of netrin-1 signal transduction depicts Rho GTPases (for review, Round and Stein, 2007) as an essential cytoplasmic signal transduction factor that regulates the actin cytoskeleton, which is involved in axon guidance, in cell-cell interactions, and cell movements in general (for review, Etienne-Manneville and Hall, 2002). Since Rho GTPase is identified from $H$. pulcherrimus (accession number, BAA75688), it would be possible to test whether HpNetrin expressed from mesenchyme blastula stage to gastrula stage plays a role in mesenchyme cell migration as has been reported in distal tip cell migration in C. elegans(e.g. Lehmann, 2001). However, the present study showed that in the MASO-injected plutei, spiculogenesis occurred was not apparently affected by deprivation of normal HpNetrin expression pattern. This apparently indicates that assumed $\mathrm{HpNetrin}$ function as a cue for primary mesenchyme cell migration (a prerequisite for normal spiculogenesis) is ruled out.

In swimming blastulae, however, no such migrating cells are formed yet, and instead, it only contains a single epithelial celllayer. HpNetrin in these embryos, thus, could be involved in epithelial cell adhesion. The present mini-blastulae that were lined with $\mathrm{HpNetrin}$ on the inner surface (basal surface) of the cavity (Fig. 
9), and lack of HpNetrin expression among non-aggregated, single embryonic cells (Fig. 8C) may support this interpretation. However, participation of Netrin-1 in epithelial morphogenesis has been known and its receptors appear to be distinctive from those involved in axon guidance, such as Unc5 and DCC (Matilainnen et al., 2007). Netrin also is involved in cell adhesion, such as reported in the formation of tubular bi-layer structures of mammary ducts in concert with Slit, a netrin receptor (Strickland, et al., 2006). According to the NCBI gene database, a slitgene was partially sequenced in $S$. purpuratus. Functional analysis of sea urchin slithas yet to be reported. However, in MASO-injected plutei, their overall morphology was not obviously disturbed, indicating ectodermal cell adhesion function may not be carried out by HpNetrin.

The present artificial embryonic cell dissociation at 20-hpf gastrula stage did not disrupt Hpnetrin transcription and protein translation in these cells that formed mini-blastulae (Fig.8), indicating that the physically intact structure of embryo or larva is not required to express the protein. Interestingly, however, miniblastulae showed closely associated two immuno-positive bands (Fig. 8B, lanes 1 and 2) that were never seen in intact larvae (Figs. 4,5). Thus, HpNetrin expression mechanism in mini-blastula may be a little different from intact larvae, such as occurrence of alternative splicing. Striking WMIHC observation in the mini-blastulae was the well-retained topological relationship between the site of $\mathrm{HpNetrin}$ expression and the direction of the serotonergic axon extension. HpNetrin lined the basal surface of the mini-blastulae, and while serotonergic cell bodies were located in the wall, their axons were restricted to inside of these mini-blastulae. These axons never extended to the apical surface of the mini-blastulae where $\mathrm{HpNetrin}$ is not expressed, indicating these axons were attracted by HpNetrin on the basal surface of the wall.

Serotonergic cells were initially formed around the HpNetrin expressing animal plate in 24-hpf prism larvae. The immunohistochemical signal of $\mathrm{HpNetrin}$ appeared to form a gradient, from the highest signal at the middle-ridge and decreasingly to the periphery of the region (Fig. 5E). Thus, the direction of initial serotonergic axon extension points to the HpNetrin-rich middle-ridge (Fig. 6A) or DMB (Fig. 6I). No axons were extended ipsilaterally toward the HpNetrin-poor region in the present study. In 53-hpf plutei, however, the growth cone of serotonergic axons crossed the HpNetrinrich middle-ridge to the contralateral ectoderm (Fig. 6G). Serotonergic axons, thus, are attracted to the HpNetrin-rich region before crossing the middle-ridge and then repulsively after crossing the region. This is supported by the present observation that serotonergic axons totally failed to extend toward the middle-ridge in Hpnetrin MASO-injected larvae that failed to form regional $\mathrm{HpNetrin}$ intensive areas, such as area-II (Fig. 10A).

Since the middle-ridge of the animal plate is the anterior end of DMB (Fig. 7), the present serotonergic axon response should be generalized to " from initial attractive and subsequent repulsive to HpNetrin". This behavior resembles the classic bi-functional cues of netrins (e.g. Dickson, 2002; Chilton, 2006; Round and Stein, 2007), such as reported in commissural axons crossing the midline in vertebrates and invertebrates (e.g. Dickson, 2002).

Alteration of axonal sensitivity to netrins is regulated by the protein receptors, such as DCC (deleted in colorectal carcinoma) and Unc-5 for instance (e.g. Dickson, 2002; Round and Stein, 2007). These receptors may play a major role in the formation of the sea urchin serotonergic nervous system, as they are predicted by the Sea Urchin Genome Project; Unc-5 (Sp-Unc5, SPU_010776) and DCC (Sp-DCC/neogenin-like, SPU_025975, 025502, 0010851) homologs (Whittaker, et al., 2006). The present study, thus, provides a first step to explore the netrin-mediated axon guidance mechanism in echinoderm nervous system formation.

\section{Materials and Methods}

\section{Animals}

Gametes of the sea urchin, $H$. pulcherrimus, were collected by blastocoelic injection of $0.5 \mathrm{M} \mathrm{KCl}$, and eggs were then inseminated. Fertilized eggs were incubated in filtered sea water (FSW) on a gyratory shaker at 18 ${ }^{\circ} \mathrm{C}$ until appropriate developmental stages described in the text.

\section{Cloning of Hpnetrin and sequencing}

Total RNA for cDNA preparation was obtained from 24 hours postfertilization (24-hpf) prism larvae with ISOGEN (NIPPON GENE, Tokyo, Japan), and was used for PCR with Super Script II and Oligo-d(T) primers (both from Invitrogen, Tokyo, Japan). Four degenerate primers were prepared according to the amino acid sequences of netrin-1s in human (U75586), mouse (U65418), Xenopus(AF033341), chicken (L34549), and zebrafish (AF017420) as follows.

F1: MGNTGYAARCCNTTYCAYTAY

F2: TTYCAYTAYGAYMGNCCNTGG

R1: YTGYTGRTANCCYTTNGCRCA

R2: YTTNGCRCANCKRTTRCANGT

$F 1$ and R1 were used for the first PCR, and F2 and R2 were for the nested $\mathrm{PCR}$ with the first PCR product template. After agarose gel electrophoresis, a single band around $1.5 \mathrm{kbps}$ was cut from the gel, and ligated to pGEMT Easy Vector (Promega, Madison, WI, USA). Sequencing was conducted by using a Big Dye terminator cycle sequencing kit with a DNA sequencer model 310 (PE Applied Biosystems, Tokyo, Japan). Based on this partial sequence, primers were designed as follows:

Net 3'-1: ATGCCGGTTTAACATGGAGCT

Net 5'-1: GCATTCGTTGGCTTCTTTTGA

Net 3'-2: ATCAGTCTAAACCCATCACGC

Net 5'-2: TTCTTTTGATGTGGCCCTGGC

5' and 3' termini of the cDNA (Hpnetrin) were obtained with Net 5'-1 and Oligo-d(T) primers combined with adaptor sequence (Invitrogen). Net 5'-2 for 5' termini and Net 3'-1 and Net 3'-2 for 3' termini were used for the first and the second PCR with the Adaptor primer (Invitrogen), respectively. The phylogenetic analysis of HpNetrin was conducted by TreeView X (Page, 1996) using netrin of Strongylocentrotus purpuratus (XP 001177385), netrin-1 of human (NP_004813), netrin-1 of Gallus gallus (NP_990750), UNC-6 of Caenorhabditis elegans (AAA80449), netrin-3 of human (netrin2 like) (NP_006172), netrin-A of Drosophila melanogaster (AAB17533), netrin-B (NP_511155), netrin-4 of human (NP_067052), human netrin-G1 (NP_055732), and -G2 (NP_115925), netrin of Ciona intestinalis (NP_001027598), netrin-1 of Xenopus tropicalis (NP_001072385), netrin1 of Mus musculus. (NP_032770), amphinetrin of Branchiostoma floridae (CAB72422), netrin precursor of Hirudo medicinalis (AAC83376), netrin1of planaria, Schmidtea mediterranea (AAY23350), netrin of Molgula tectiformis (BAF35976), and netrin-1 of hemichordate Saccoglossus kowalevskii (ABD97277).

\section{Reverse transcriptase (RT)-PCR analysis}

The chronological mRNA accumulation levels of Hpnetrin was examined using $1 \mu \mathrm{g}$ cDNA templates prepared from unfertilized eggs, eggs 30 minutes after insemination, 5-hpf morulae, 15-hpf swimming blastulae, 16hpf mesenchyme blastulae, 18-hpf early gastrulae, 24-hpf prism and 48-hpf plutei, by PCR using the following primer set:

Forward: TTGTGGTGGTACGTGGTTAG,

Reverse: CTCGTTGGAATTTCCGCATC. Potential contamination by DNA in amplimers was examined by PCR without reverse transcriptase at each 
developmental stage. Positive controls were carried out with $1 \mu \mathrm{g}$ cDNA template using $H$. pulcherrimus ubiquitin (Hpubr) primer set: Hpubi-F: GAGCTGCGATGTATTTGCCAGAYG, Hpubi-R: TTTGATGGAATAACAAATAACYGATTGCTT (Nemer et al., 1991).

\section{Antibody generation}

To raise antibodies (Ab) against $\mathrm{HpNetrin,} \mathrm{a} \mathrm{14-amino} \mathrm{acid} \mathrm{sequence}$ (from Leu ${ }_{600}$ to $\mathrm{Phe}_{613}$ ) near the C-terminal end of the $\mathrm{C}$ domain (Fig. 1, underline) was chosen by referring to the results of Physico-Chemical Profile analysis by Network Protein Sequence Analysis (http://npsapbil.ibcp.fr/cgi-bin/npsa_automat.pl?page=npsa_pcprof.html). According to a BLAST Search the sequence was netrin specific. The synthetic peptide was tagged with Limulus Keyhole Limpet Hemocyanin (KLH) protein, and injected into mice. The $A b$ was recovered as ascites obtained by intraperitoneal injection of $1 \mathrm{ml}$ of $1 \times 10^{7}$ cells $/ \mathrm{ml} \mathrm{SP} 2$ myeloma (c.f. Katow et al., 2004). The immunospecificity was examined with immunoblotting by applying ascites 1:1000 diluted in TBST (500mM Tris- $\mathrm{HCl}(\mathrm{pH} 7.0), 0.15 \mathrm{M}$ $\mathrm{NaCl}$ and $0.05 \%$ Tween-20) with or without $1 \mathrm{mg} / \mathrm{ml}$ of the synthetic antigen peptide onto whole embryo lysate. The sample was prepared from 18-hpf early gastrulae, and blotted to nitrocellulose filters according to the method of Towbin et al. (1979) after 10\% sodium dodecyl sulfate-acrylamide gel electrophoresis (SDS-PAGE) under reducing condition. Further detail of the technique is described in the following "Immunoblotting" section. For WMIHC examination of the Ab, 16-hpf mesenchyme blastulae fixed with $4 \%$ paraformaldehyde were treated with Abs diluted 1:50 in PBST (0.10M phosphate-buffered saline with $0.05 \%$ Tween 20 ) with or without $3 \mathrm{mg} / \mathrm{ml}$ synthetic antigen peptide. The primary Ab was detected with Alexa Fluor 594-tagged goat anti-mouse IgG $(\mathrm{H}+\mathrm{L})$ Abs (Molecular Probes Inc., Eugene, OR), examined under a Nikon epi-fluorescent microscope (Nikon Corporation, Tokyo, Japan) or a Leitz DMR fluorescence microscope (Leica Microsystems Co. Ltd., Tokyo, Japan), and photographed with an Olympus Camedia C-3030ZOOM digital camera (Olympus Optical Co. Ltd., Tokyo, Japan). The data were imported into Photoshop CS2 v.9.02 image software (Adobe), and merged the double stained images and light field microgram images for analysis.

\section{Immunoblotting}

Unfertilized eggs, eggs 30 minutes after fertilization as well as embryos and larvae (5-hpf morula, 15-hpf swimming blastula, 16-hpf mesenchyme blastula, 18-hpf early gastrula, 20-hpf mid-gastrula, 24-hpf prism and 48hpf pluteus stages) were solubilized, respectively, in a lysis buffer (6 M urea, $1 \%$ Nonidet P-40, $10 \mathrm{mM}$ Tris- $\mathrm{HCl}, \mathrm{pH} 7.6$ ). These samples were ethanol precipitated by adding final $75 \%$ of cold $100 \%$ ethanol (stored at $-30^{\circ} \mathrm{C}$ until immediately before use), and stored a few days at $-20^{\circ} \mathrm{C}$, and vacuum dried after dehydration with cold pure ethanol. These dried samples (powder sample) were separated on $10 \%$ SDS-PAGE slab gels under reducing condition according to the method of Laemmli (1970), and electrophoretically transferred to nitrocellulose filters according to Towbin et al. (1979) at $400 \mathrm{~mA}$ for 2 hours at $4^{\circ} \mathrm{C}$. The nitrocellulose filters were treated with $5 \%$ skim milk in TBST for 1 hour to block non-specific binding. The blots were incubated with $70 \mu \mathrm{g} / \mathrm{ml}$ of anti-HpNetrin Ab that was purified using a Melon Gel IgG Spin Purification Kit (Pierce, Rockford, IL, USA), and then with alkaline phosphatase-tagged anti-mouse IgG goat Ab (Sigma-Aldrich Co., St. Louis, MO, USA) 1:30,000 diluted in TBST. The immunoreaction was visualized with nitrobluetetrazolium/5-bromo-4-chloro-3-indolyl phosphate (NBT/BCIP, Promega, Madison, WI. USA) according to the manufacturer's protocol.

\section{Whole mount immunohistochemistry (WMIHC)}

Embryos at 15-hpf swimming and 16-hpf mesenchyme blastula stages, 18-hpf early and 23-hpf late gastrula stages, and larvae at 24-hpf prism and 48-hpf pluteus stages were fixed with $4 \%$ paraformaldehyde diluted in FSW for 20 min, dehydrated through increasing concentration of ethanol from $30 \%$ to $70 \%$, and stored in $70 \%$ ethanol at $4^{\circ} \mathrm{C}$ until use. The samples were hydrated to PBST, pre-incubated for 30 minutes with $1 \%$ bovine serum albumin (Sigma-Aldrich Co.) to block non-specific binding, and incubated with anti-HpNetrin Ab alone (1:50 diluted in PBST) two nights or it was followed by double staining with rabbit anti-serotonin Abs (Sigma-Aldrich Co., 1:500 diluted in PBST) over night at $4^{\circ} \mathrm{C}$. The immunoreaction was visualized with secondary Abs, Alexa Fluor 594-tagged anti-mouse IgG goat Abs for HpNetrin and Alexa Fluor 488-tagged anti-rabbit IgG goat Abs for serotonin. Both secondary Abs were from Molecular Probes Inc., and were diluted 1:500 in PBST. Both single stained and double stained images were examined with a fluorescent microscope, photographed as stated above, and their images were analyzed with respective light field images by Photoshop CS2 v.9.02 software (Adobe) for image analysis as described above. Samples were examined also under a Micro-Radiance Confocal laser scanning microscope (Bio-Rad, Hercules, USA) with optical section of $1 \mu \mathrm{m}$, and analyzed with ImageJ $1.37 \mathrm{v}$, a public domain from $\mathrm{NIH}$, and Photoshop software.

\section{Cell culture}

Embryos at the 20-hpf mid-gastrula stage were incubated once with calcium-magnesium-free artificial sea water for 10 minutes, and were replaced and incubated with calcium-free artificial sea water (CFASW) for 10 minutes. They were manually shaken in fresh CFASW to dissociate the embryos and were examined microscopically. The dissociated embryonic cells were further filtered with $20 \mu \mathrm{m}$ Nitex Bolting Cloth (Wildlife Supply Co., Buffalo, NY, USA) to obtain a suspension of single cells, and washed once with fresh FSW that contained $200 \mathrm{unit} / \mathrm{ml}$ penicillin $\mathrm{G}$ and $200 \mu \mathrm{g} / \mathrm{ml}$ streptomycin sulfate (Both from Gibco BRL). Cells were then incubated at $1.5 \times 10^{7} \mathrm{cells} / \mathrm{ml}$ on 24 -well plates on a rotary shaker at $110 \mathrm{rpm}$ at $18^{\circ} \mathrm{C}$ until intact embryos reached 48 -hpf pluteus stage. For WMIHC, re-aggregated cells were fixed with $4 \%$ paraformaldehyde, washed three times with PBST, and treated with Abs as stated above. For immunoblotting, an aliquot of reaggregated cells and 48-hpf plutei were dissolved in SDS-PAGE sample buffer, respectively. Their protein contents were examined at $A_{280}$, and loaded each sample at $5 \mu \mathrm{g} /$ well for $10 \%$ SDS-PAGE. These samples were examined as stated above. For RT-PCR analysis, cDNA was prepared from 20-hpf mid-gastrulae, dispersed cells immediately after dissociation, and cell-aggregates at 24 hours after the incubation in vitroas stated above. RTPCR was performed as stated above. WMIHC was performed onto cellaggregates after double staining with anti-serotonin Abs and anti-HpNetrin $\mathrm{Ab}$, and examined under a fluorescent microscope as stated above.

\section{Microinjection of morpholino anti-sense oligonucleotide (MASO)}

Unfertilized eggs were fixed between cover slip and glass slide, and microinjected with 10,12 , or $20 \mu \mathrm{M}$ of Hpnetrin MASO: 5'GACTCACAATTTGCATTTTACCTTC3', or its standard control oligonucleotide: 5'CCTCTTACCTCAGTTACAATTTATA3', in double distilled water. Then the eggs were inseminated, and incubated for 56 hours in 24well plate at $18^{\circ} \mathrm{C}$. Both oligonucleotides were purchased from Gene Tool (Eugene, OR, USA). Then larvae were fixed and double stained with antiserotonin $\mathrm{Ab}$ and anti-HpNetrin $\mathrm{Ab}$, and examined under a confocal laser scanning microscopy as stated above.

\section{Acknowledgements}

I thank H. Yasuda for supporting sequencing Hpnetrin, Dr. K. Kyozuka for MASO microinjection, and M. Washio, Research Center for Marine Biology, for collecting sea urchins used in the present study and for preparing myeloma cells for raising ascites. I also thank Dr. Gary Wessel, Brown University, for critically reading this manuscript.

\section{References}

AUGSBURGER, A., SCHUCHARDT, A., HOSKINS, S., DODD, J., AND BUTLER, S (1999) BMPs as mediators of roof plate repulsion of commissural neurons. Neuron 24: $127-141$

BOSSING, T., AND BRAND, A.H. (2006) Determination of cell fate along the 
anterior axis of the Drosophila ventral midline. Development 133: 1001-1012.

BURKE, R.D., ANGERER,L.M., ELPHICK, M.R., HUMPHREY, G.W., YAGUCHI, S., KIYAMA, T., LIANG, S., MU, X., AGCA, C., KLEIN, W.H., BRANDHORST, B.P., ROWE, M., WILSON, K., CHURCHER,A.M., TAYLOR, J.S., CHEN, N., MURRAY, G., WANG, D., MELLOTT, D., OLINSKI, R., HALLBÖÖK, F., AND THORNDYKE, M.C. (2006) A genomic view of the sea urchin nervous system. Dev Bio/300: 434-460.

BYRNE, M., NAKAJIMA, Y., CHEE, F.C., AND BURKE, R.D. (2007) Apical organs in echinoderm larvae: insights into larval evolution in the Ambulacraria. Evo Dev 9: $432-445$

CHARRON, F., STEIN, E., JEONG, J., MCMAHON, A.P., AND TESSIER-LAVIGNE, M. (2003) The morphogen sonic hedgehog is an axonal chemoattractant that collaborates with netrin-1 in midline axon guidance. Cel/113: 11-23.

CHILTON, J.K. (2006) Molecular mechanisms of axon guidance. Dev Bio/292: 1324.

CHISHOLM, A., AND TESSIER-LAVIGNE, M. (1999) Conservation and divergence of axon guidance mechanisms. Curr Opin Neurobio/9: 603-615.

DE LA TORRE, J.R., HÖPKER, V.H., MING, G.-I., POO, M., TESSIER-LAVIGNE, M., HEMMATI-BRIVANLOU, A., AND HOLT, C.E. (1997) Turning of retinal growth cones in a netrin-1 gradient mediated by the netrin receptor DCC. Neuron 19:1211-1224.

DEINER, M.S., KENNEDY, T.E., SERAFINI, T., TESSEIR-LAVIGNE, M., AND SRETAVAN, D.W. (1997) Netrin-1 and DCC mediate axon guidance locally at the optic disc: Loss of function leads to optic nerve hypoplasia. Neuron 19: 575 589.

DICKSON, B.J. (2002) Molecular mechanism of axon guidance. Science 298: 1959-1964.

ETIENNE-MANNEVILLE, S., AND HALL, A. (2002) Rho GTPases in cell biology. Nature 420: 629-635

GEISBRECHT, B.V., DOWD, K.A., BARFIELD, R.W., LONGO, P.A., AND LEAHYS, D.J. (2003) Netrin binds discrete subdomains of DCC and UNC5 and mediates interactions between DCC and heparin. J Biol Chem 278: 32561-32568.

GUAN, W., AND CONDIC, M.L. (2003) Characterization of netrin-1, neogenin and cUNC-5H3 expression during chick dorsal root ganglia development. Gene Expr Patterns 3:369-373.

HALL, A.C., LUCAS, F.R., AND SALINAS, P.C. (2000) Axonal remodeling and synaptic differentiation in the cerebellum is regulated by WNT-7a signaling. Ce/l 100: 525-535.

HIRTH, F., AND REICHERT, H. (2007) Basic nervous system types: One or many? In "Evolution of Nervous Systems" Elsevier Inc. pp. 55-72.

KAMMERMEIER, L., AND REICHERT, H. (2001) Common developmental genetic mechanism for patterning invertebrate and vertebrate brain. Brain Research Bulletin 55: 675-682.

KATOW, H., YAGUCHI, S., KIYOMOTO, M., AND WASHIO, M. (2004) The 5-HT receptor cell is a new member of secondary mesenchyme cell descendants and form a major blastocoelar network in sea urchin larvae. Mech Dev121: 325-337.

KATOW, H., YAGUCHI, S., KYOZUKA, K. (2007) Serotonin stimulates $\left[\mathrm{Ca}^{2+}\right.$ ] elevation in ciliary ectodermal cells of echinoplutei through a serotonin receptor cell network in the blastocoel. J Exp Bio/210: 403-412.

KEARNEY, J.B., WHEELER, S.R., ESTES, P., PARENTE, B., AND CREWS, S.T. (2004) Gene expression profiling of the developing Drosophila CNS midline cells. Dev Bio/275: 473-492.

KENNEDY, T.E., SERAFINI, T., DE LA TORRE, J.R., AND TESSIER-LAVIGNE, M. (1994) Netrins are diffusible chemotrophic factors for commissural axons in the embryonic spinal cord. Cel/78: 425-435

KELEMAN, K., AND DICKSON, B.J. (2001) Short- and long-range repulsion by the Drosophila Unc5 netrin receptor. Neuron 32: 605-617.

KOCH, M., MURRELL, J.R., HUNTER, D.D., OLSON, P.F., JIN, W., KEENE, D.R., BRUNKEN, W.J., AND BURGESON, R.E. (2000) A novel member of the netrin family, $\beta$-netrin, shares homology with the $\beta$ chain of laminin: Identification, expression, and function characterization. J Cel/ Bio/151: 221-234.

LAEMMLI, U.K. (1970) Cleavage of structural proteins during the assembly of the bacteriophage T4. Nature 227: 680-685.

LEE, C.M., YU, D.S., CREWS, S.T., and KIM, S.H. (1999). The CNS midline cells and spitz class genes are required for proper patterning of Drosophila ventral neuroectoderm. Int J Dev Bio/43: 305-315.

LEHMANN, R. (2001) Cell migration in invertebrates: cues from border and distal tip cells. Curr Opin Genet Dev11: 457-463.

LEONARDO, E.D., HINCK, L., MASU, M., KEINO-MASU, K., ACKERMAN, S.L., AND TESSIER-LAVIGNE, M. (1997) Vertebrate homologues of C. elegans UNC-5 are candidate netrin receptor. Nature 386: 833-838.

LICHTNECKERT, R., AND REICHERT, H. (2007) Origin and evolution of the nervous system. In "Evolution of Nervous System" pp 289-315. Elsevier Inc.

LIM,Y.-S., AND WADSWORTH, W.G. (2002) Identification of domains of netrin UNC-6 that mediate attractive and repulsive guidance and responses from cells and growth cones. JNeurosci22: 7080-7087.

MANITT, C., COLICOS, M.A., THOMPSON, K.M., ROUSSELLE, E., PETERSON, A.C., AND KENNEDY. T.E. (2001) Widespread expression of netrin-1 by neurons and oligodendrocytes in the adult mammalian spinal cord. JNeurosci 21:3911-3922

MANITT, C., AND KENNEDY, T.E. (2002) Where rubber meets the road: Netrin expression and function in developing and adult nervous system. Prog Brain Res 137: 425-442.

MANN, F., HARRIS, W.A., AND HOLT, C.E. (2004) New views on retinal axon development: a navigation guide. Int. J. Dev. Biol. 48:957-64

MATILAINEN, T., HAUGAS, M., KREIDBERG, JA. AND SALMINEN, M. (2007) Analysis of Netrin 1 receptors during inner ear development. Int. J. Dev. Biol. 51:409-413

MÜLLER, B.K., BONHOEFFER, F., AND DRESCHER, U. (1996) Novel gene families involved in neural pathfinding. Curr Opin Genet Dev 6: 469-474.

NAKAJIMA, Y., BURKE, R.D., AND NODA, Y. (1993) The structure and development of the apical ganglion in the sea urchin pluteus larvae of Strongylocentrotus droebachiensis and Mespilia globules. Dev Growth Differ 35: 531-538.

NAKAJIMA, Y., KANEKO, H., MURRAY, G., AND BURKE, R.D. (2004) Divergent patterns of neural development in larval echinoids and asteroids. Evo Dev6: 95104.

NEMER, M., RONDINELLI, E., INFANTE, D., AND INFANTE, A.A. (1991) Polyubiquitin RNA characteristics and conditional induction in sea urchin embryos. Dev Biol145: 255-265.

PAGE, R.D.W. (1996) TREEVIEW: An application to display phylogenetic trees on personal computers. Computer Applications in the Biosciences 12: 357-358.

ROUND, J., AND STEIN, E. (2007) Netrin signaling leading to direct growth cone steering. Curr Opin Neurbio/ 17:15-21.

SCHWARTING, G.A., RAITCHEVA, D., BLESS, E.P., ACKERMAN, S.L., AND TOBET, S. (2004) Netrin 1-mediated chemoattraction regulates the migratory pathway of LHRH neurons. Eur JNeurosci19:11-20.

SERAFINI, T., KENNEDY, T.E., GALKO, M.J., MIRZAYAN, C., JESSELL, T.M., AND TESSIER-LAVIGNE, M. (1994) The netrins define a family of axon outgrowth-promoting proteins homologous to C. elegans UNC-6. Cel/78: 409424.

STOECKLI, E.T., and LANDMESSER, L.T. (1998). Axon guidance at choice points. Curr Opin Neuro/8:73-79.

STRICKLAND, P., SHIN, G.C., PLUMP, A., TESSIER-LAVIGNE, M., AND HINCK, L. (2006) Slit2 and netrin 1 act synergistically as adhesive cues to generate tubular bi-layers during ductal morphogenesis. Development 133: 823-32.

TESSIER-LAVIGNE, M., AND GOODMAN, C.S. (1996) The molecular biology of axon guidance. Science 274: 1123-1133.

TOWBIN, H., STAEHEKIN, T., AND GORDEON, J. (1979) Electrophoretic transfer of proteins from acrylamide gels to nitrocellulose sheets: procedure and application. Proc Natl Acad Sci USA 76: 4350-4354.

TSAI, H.-H., TESSIER-LAVIGNE, M., MILLER, R.H. (2003) Netrin 1 mediates spinal cord oligodendrocyte precursor dispersal. Development 130:2095-2105.

WANG,Y., THEKDI, N., SMALLWOOD, P.M,. MACKE, J.P, AND NATHANS, J. (2002) Frizzled-3 is required for the development of major fiber tracts in the rostral CAN. J Neurosci22: 8563-8573.

WHEELER, S.R., KEARNEY, J.B., GUARDIOLA, A.R., AND CREWS, S.T. (2006) Single-cell mapping of neural and glial gene expression in the developing Drosophila CNS midline cells. Dev Bio/294: 509-524.

WHITTAKER, C.A., BERGERON, K.-F., WHITTLE, J., BRANDHORST, B.P., BURKE, R.D., AND HYNES, R.O. (2006) The echinoderm adhersome. Dev Biol 
300: $252-266$.

YAGUCHI, S., KANOH, K., AMEMIYA, S., AND KATOW, H. (2000) Initial analysis of immunochemical cell surface properties, location and formation of the serotonergic apical ganglion in sea urchin embryos. Dev Growth Differ42: 479488.
YAGUCHI, S., AND KATOW, H. (2003) Expression of tryptophan 5-hydroxylase gene during sea urchin neurogenesis and role of serotonergic nervous system in larval behavior. J Comp Neuro/466: 219-229.

ZALLEN, J.A., KIRCH, S.A., AND BARGMANN, C.I. (1999) Genes required for pathfinding and extension in the C. elegansnerve ring. Development126:36793692 .

\section{Further Related Reading, published previously in the Int. J. Dev. Biol.}

See our recent Special Issue Fertilization, in honor of David L. Garbers and edited by Paul M. Wassarman and Victor D. Vacquier at: http://www.ijdb.ehu.es/web/contents.php?vol=52\&issue=5-6

See our recent Special Issue Ear Development edited by Fernando Giraldez and Bernd Fritzsch at: http://www.ijdb.ehu.es/web/contents.php?vol=51\&issue=6-7

Analysis of Netrin 1 receptors during inner ear development Tanja Matilainen, Maarja Haugas, Jordan A. Kreidberg and Marjo Salminen Int. J. Dev. Biol. (2007) 51: 409-414

Serotonin involvement in the metamorphosis of the hydroid Eudendrium racemosum Giuliana Zega, Roberta Pennati, Arianna Fanzago and Fiorenza De Bernardi Int. J. Dev. Biol. (2007) 51: 307-313

Axon guidance in the inner ear Donna M. Fekete and Andrea M. Campero Int. J. Dev. Biol. (2007) 51: 549-556

New views on retinal axon development: a navigation guide Fanny Mann, William A. Harris and Christine E. Holt Int. J. Dev. Biol. (2004) 48: 957-964

Multiple axon guidance cues establish the olfactory topographic map: how do these cues interact?

James A St John, Heidi J Clarris and Brian Key

Int. J. Dev. Biol. (2002) 46: 639-647

Homeotic genes influence the axonal pathway of a Drosophila embryonic sensory neuron.

David J Merritt and Paul M Whitington

Int. J. Dev. Biol. (2002) 46: 633-638

Axon guidance receptors direct growth cone pathfinding: rivalry at the leading edge.

Helen M Cooper

Int. J. Dev. Biol. (2002) 46: 621-631

Development of axon pathways in the zebrafish central nervous system. Jensen Hjorth and Brian Key Int. J. Dev. Biol. (2002) 46: 609-619

Orienting axon growth: spinal nerve segmentation and surround-repulsion. D Tannahill, J M Britto, M M Vermeren, K Ohta, G M Cook and R J Keynes Int. J. Dev. Biol. (2000) 44: 119-127

Action of serotonin antagonists on cytoplasmic calcium levels in early embryos of sea urchin Lytechinus pictus.

Y B Shmukler, G A Buznikov and M J Whitaker Int. J. Dev. Biol. (1999) 43: 179-182

2006 ISI ** Impact Factor $=3.577^{\star *}$

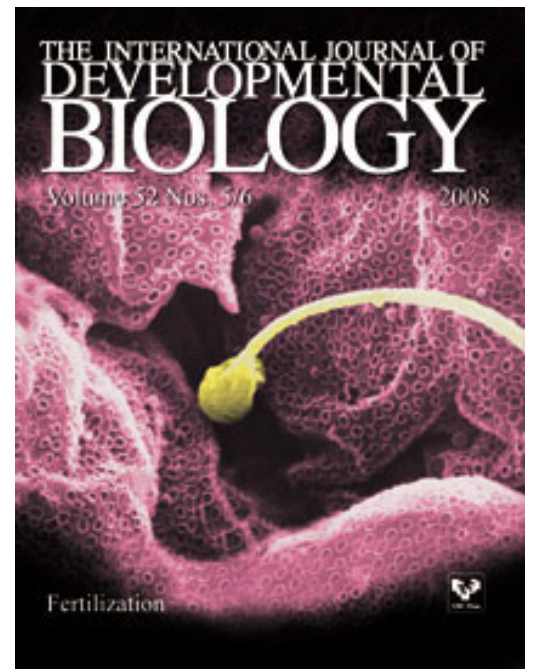

